\title{
Efecto de la urea y del tiempo en la fermentación en estado sólido de la harina de frutos del árbol del pan (Artocarpus altilis)
}

\author{
The urea and time effect in the fermentation in \\ the solid state of the fruit's flour of the bread tree \\ (Artocarpus altilis)
}

Fecha de recepción: 17 de febrero de 2015 Fecha de aprobación: 23 de mayo de 2015
Odelin Brea-Maure', Arabel Elías-lglesias² Abel Ortiz-Milán ${ }^{3}$, Walter Motta-Ferreira ${ }^{4}$ Suset Hechavarría-Riviaux ${ }^{5}$

\section{Resumen}

Para evaluar el efecto de la inclusión de niveles de urea y del tiempo sobre los indicadores fermentativos y químicos de la harina de frutos del árbol del pan, fermentada en estado sólido, se mezcló homogéneamente con esta, sulfato de magnesio $(0,2 \%)$, premezcla mineral $(0,5 \%)$ y agua destilada. Se utilizó un diseño completamente aleatorizado con arreglo factorial $(4 \times 4)$, con 4 repeticiones por tratamiento para evaluar el efecto de la urea $(0 ; 0,5 ; 1,0$ y 1,5\%) y cuatro tiempos de fermentación $(0,12,24$ y 48 h). En el estudio, a medida que se incrementa el nivel de urea, aumenta la concentración de amoníaco y, por ende, el pH, lo que depende, en menor o mayor grado, de la concentración de los ácidos orgánicos (AGCC y láctico). Al final del proceso fermentativo hubo una disminución en la MS $(\mathrm{P}<0,001)$ a medida que transcurrió el tiempo de fermentación. La adición de 1,5\% de urea produjo un incremento en la concentración de PB a las 24 y $48 \mathrm{~h}$ sin diferencia entre ellos; algo similar ocurrió con la PV y la relación entre ellos (PV:PB)*100 sin interacción entre los factores en estudios. A las $48 \mathrm{~h}$ hubo un aumento mayor de 2,15 unidades porcentuales en la PB con respecto a la hora cero. Se concluye que la adición de urea en el proceso de fermentación de la harina de frutos del árbol del pan en estado sólido incrementa la síntesis de proteína microbiana.

Palabras clave: Árbol del pan, fermentación en estado sólido, harina del árbol del pan.

1 Ph. D. Universidad de Guantánamo (Guantánamo, Cuba). odelinbm@cug.co.cu.

2 Ph. D. Instituto de Ciencia Animal (Mayabeque. Cuba). aelias@ica.co.cu.

3 Ph. D. Universidad de Guantánamo (Guantánamo, Cuba).

4 Ph. D. Universidade Federal de Minas Gerais (Belo Horizonte, Brasil).

5 Ph. D. Universidad de Guantánamo (Guantánamo, Cuba). 


\begin{abstract}
To evaluate the urea levels inclusion effect and the time on the fermentative and chemical indicators of the flour of fruits of the bread tree, fermented in solid state, was mixed homogeneously, with sulfate of magnesium $(0,2 \%)$, mineral premix $(0,5 \%)$ and diluted in distilled water. A totally randomized design was used with factorial arrangement $(4 \times 4)$ with 4 repetitions for treatment to evaluate the effect of the urea $(0$, $0,5,1,0$ and $1,5 \%)$ and four times of fermentation $(0,12,24$ and $48 \mathrm{~h})$.
\end{abstract}

In the study, as the level of urea is increased, the concentration of ammonia grows the $\mathrm{pH}$, which depends, in smaller or bigger degree, on the organic acids concentration (AGCC and lactic). At the end of the fermentative process there was a decrease in the MS $(P<0,001)$ as the fermentation time passed. The addition of $1,5 \%$ of urea produced an increment in the concentration from PB at $2448 \mathrm{~h}$ without difference among them, something similar happened with the PV and the relationship among them (PV:PB)*100 without interaction among the factors in study.

At $48 \mathrm{~h}$ there was an increase bigger than 2,15 percentage units in the PB with regard to the zero hour. It is concluded that the addition of urea in the fermentation process of the flour from the fruits of the bread tree in solid state, increases the microbial protein synthesis.

Key words: bread tree, fermentation in solid state, flour from the bread tree. 


\section{Introducción}

En Cuba, específicamente en la provincia de Guantánamo, es posible aprovechar en mayor escala los beneficios del fruto del árbol del pan (Artocarpus altilis), el cual es utilizado empíricamente por los campesinos como alimento animal, pero más del 60\% (30 000 toneladas) de la producción se pierde en los campos (1).

Los procesos fermentativos permiten mejorar la composición química de algunos productos agrícolas y obtener nuevas opciones para la alimentación animal. La harina del fruto del árbol del pan, a pesar de contener bajos niveles de proteína bruta y presentar metabolitos secundarios, como saponinas, según Leyva (2), constituye una fuente importante de energía y vitaminas. En la actualidad se desarrollan diversas investigaciones para incrementar el valor nutricional y reducir las limitantes nutricionales de diferentes productos; una alternativa es por vía de la fermentación, a través de la cual se pueden obtener alimentos energético-proteicos biotransformados en sus diferentes variantes, donde se incluyen fuentes energéticas y proteínicas para mejorar la calidad y digestibilidad del producto que se fermenta (3, 4, 5, 6 y 7 ).

El objetivo del trabajo fue evaluar el efecto de inclusión de niveles de urea y el tiempo sobre los indicadores fermentativos y químicos de la harina de frutos del árbol del pan fermentada en estado sólido.

\section{Materiales y métodos}

Los frutos se cosecharon en el municipio Yateras, provincia Guantánamo, el cual tiene una topografía montañosa casi en un $100 \%$, con temperatura promedio anual de $34{ }^{\circ} \mathrm{C}$ y un promedio histórico anual de precipitaciones de $1882 \mathrm{~mm}$. La harina de frutos del árbol del pan se elaboró según la metodología descrita por Leyva y Valdivié (8). El experimento se realizó en el laboratorio de Nutrición Animal perteneciente al departamento de Zootecnia de la Facultad de Medicina Veterinaria de la Universidad Federal de Minas Gerais, Belo Horizonte, Brasil.
Se utilizó un diseño completamente aleatorizado, con arreglo factorial $(4 \times 4)$ con 4 repeticiones. El primer factor fue el nivel de urea $(0,0,5,1,0$ y $1,5 \%$ ) y el segundo, el tiempo de fermentación $(0$, 12,24 y $48 \mathrm{~h}$ ). Se analizaron los efectos principales y las interacciones con el programa estadístico INFOSTAT (9). Se realizó un análisis de varianza simple para todas las variables, y en los casos necesarios se aplicó la dócima de comparación múltiple de Duncan (10) para $\mathrm{P}<0,05$.

La urea se diluyó en agua destilada y se ajustó la cantidad de líquido que se introdujo, para que todos los tratamientos tuvieran entre el 60 y el $70 \%$ de humedad inicial. Se mezclaron $100 \mathrm{~g}$ de muestra y se depositaron en frascos Roux, que se taparon con algodón y se incubaron a temperatura ambiente $\left(28 \pm 2{ }^{\circ} \mathrm{C}\right)$ durante 12,24 y $48 \mathrm{~h}$; cada frasco constituyó una unidad experimental.

Después de la incubación, el contenido de los frascos Roux se recolectó en su totalidad y homogeneizó individualmente. Se pesaron 10 g de cada muestra, los cuales se colocaron en Erlenmeyer de $100 \mathrm{~mL}$ y se les adicionó $90 \mathrm{~mL}$ de agua destilada estéril, agitándose durante 30 minutos con un equipo Griffin Flash Shaker. Posteriormente, se procedió a filtrar las muestras a través de gasas estériles, según metodología descrita por Elías et al. (11).

Al filtrado se le midió, inmediatamente, el $\mathrm{pH}$ con pHmetro digital (CD70) de precisión \pm 0,01 unidades. El amoníaco $\left(\mathrm{NH}_{3}\right)$ se determinó según Conway (12) y los ácidos grasos volátiles individuales de cadena corta, ácidos acéticos, se determinaron por cromatografía de gases (Gas Cromatograph, SHIMADZU, GC-17A) en muestras preparadas con ácido metafosfórico (13), y el ácido láctico según Madrid et al. (14).

El total de los sólidos que quedó en los frascos Roux se secó a $60{ }^{\circ} \mathrm{C}$ y se molió en molino de martillo marca CULAT II, con criba de $1 \mathrm{~mm}$. Para el análisis químico se determinó: materia seca (MS), materia seca residual (MSR) y proteína bruta (PB), según el método descrito por la AOAC (15); la proteína verdadera (PV), según el método de 
Berstein, citado por (16), y la relación (PV:PB)x100 se calculó según Elías y Lezcano (3).

\section{Resultados Y Discusión}

Con respecto a las variables fermentativas, hubo interacción $(P<0,001)$ entre los niveles de urea añadidos y el tiempo de fermentación en la concentración de $\mathrm{pH}$ y amoníaco y en la producción de ácidos acético y láctico (Tabla I). Al incorporarse urea al proceso, se incrementa el $\mathrm{pH}$ con respecto al control; sin embargo, a las 12 y 24 $\mathrm{h}$ no hubo diferencia entre tratamientos al añadir 0,5 y $1 \%$ de urea. Cuando se incorporó 1,5\% de urea a partir de las $48 \mathrm{~h}$ difirió con respecto a los demás tratamientos. Por otro lado, en los tres primeros niveles $(0,0,5$ y $1,0 \%)$ hubo un descenso del $\mathrm{pH}$ a las $48 \mathrm{~h}$ de fermentación. Los menores valores se obtuvieron con los niveles 0 y $0,5 \%$ de urea a las $48 \mathrm{~h}$, y los mayores, al incluir $1,5 \%$, sin diferencias en este tiempo de fermentación.

Tabla I. Efecto de niveles de urea y del tiempo de fermentación en la concentración de $\mathrm{pH}, \mathrm{NH}_{3}$ y ácidos acético y láctico durante la FES (fermentación en estado sólido) de la HFP (harina de frutos del árbol del pan)

\begin{tabular}{|c|c|c|c|c|c|c|}
\hline \multirow{2}{*}{ Indicadores } & \multirow{2}{*}{$\begin{array}{c}\text { Tiempo } \\
\text { h } \\
\end{array}$} & \multicolumn{4}{|c|}{ Niveles de urea, \% } & \multirow{2}{*}{$\begin{array}{l}\text { EE ( } \pm) \\
\text { Signif. }\end{array}$} \\
\hline & & $\mathbf{0}$ & 0,5 & 1,0 & 1,5 & \\
\hline \multirow{4}{*}{ pH } & 0 & $5,12^{\mathrm{bc}}$ & $5,48^{\text {defg }}$ & $5,63^{\text {defg }}$ & $5,74^{\mathrm{g}}$ & \multirow{3}{*}{0,09} \\
\hline & 12 & $5,35^{\mathrm{cd}}$ & $5,42^{\text {def }}$ & $5,46^{\text {defg }}$ & $5,74^{\mathrm{g}}$ & \\
\hline & 24 & $5,35^{c d}$ & $5,39^{\text {cde }}$ & $5,42^{\text {def }}$ & $5,69^{f g}$ & \\
\hline & 48 & $4,52^{\mathrm{a}}$ & $4,55^{\mathrm{a}}$ & $4,86^{b}$ & $5,66^{\mathrm{fg}}$ & $\mathrm{P}=0,0002$ \\
\hline \multirow{3}{*}{$\mathrm{NH}_{3}$} & 0 & $1,37^{a}$ & $4,12^{d}$ & $4,81^{\mathrm{e}}$ & $5,92^{\mathrm{g}}$ & \multirow{3}{*}{0,12} \\
\hline & 12 & $1,51^{\mathrm{a}}$ & $4,11^{d}$ & $4,85^{\mathrm{e}}$ & $8,14^{\mathrm{h}}$ & \\
\hline & 24 & $2,07^{b}$ & $4,21^{d}$ & $5,47^{f}$ & $8,25^{\mathrm{h}}$ & \\
\hline \multirow[t]{2}{*}{$\mathrm{meq} / \mathrm{L}$} & 48 & $2,62^{c}$ & $4,40^{d}$ & $5,48^{f}$ & $8,29^{h}$ & \multirow[t]{2}{*}{$P<0,0001$} \\
\hline & 0 & $5,96^{\mathrm{g}}$ & $5,85^{\mathrm{g}}$ & $4,23^{e}$ & $2,36^{b c}$ & \\
\hline \multirow{2}{*}{ Acético } & 12 & $5,75^{\mathrm{g}}$ & $5,23^{f}$ & $3,10^{d}$ & $2,36^{b c}$ & \multirow{2}{*}{0,15} \\
\hline & 24 & $5,23^{f}$ & $4,10^{\mathrm{e}}$ & $2,57^{c}$ & $2,03^{a b}$ & \\
\hline $\mathrm{mmol} / \mathrm{L}$ & 48 & $5,06^{f}$ & $3,98^{\mathrm{e}}$ & $2,36^{\mathrm{bc}}$ & $1,81^{\mathrm{a}}$ & $P=0,0001$ \\
\hline \multirow{3}{*}{ Láctico } & 0 & $1,18^{d}$ & $0,95^{\mathrm{ab}}$ & $0,94^{\mathrm{ab}}$ & $0,87^{a}$ & \multirow{3}{*}{0,04} \\
\hline & 12 & $1,47^{f}$ & $1,05^{\mathrm{bc}}$ & $1,03^{\text {bc }}$ & $0,95^{\mathrm{ab}}$ & \\
\hline & 24 & $1,59^{\mathrm{g}}$ & $1,11^{\mathrm{cd}}$ & $1,04^{\mathrm{bc}}$ & $0,98^{\mathrm{ab}}$ & \\
\hline \multirow[t]{2}{*}{$\mathrm{mmol} / \mathrm{L}$} & & & & & & \multirow[t]{2}{*}{$P=0,0046$} \\
\hline & 48 & $1,69^{\mathrm{g}}$ & $1,36^{\mathrm{e}}$ & $1,32^{\mathrm{e}}$ & $1,17^{\mathrm{d}}$ & \\
\hline
\end{tabular}

$a, b, c, d, e, f, g, h$

Medias con letras distintas indican diferencias a $\mathrm{P}<0,05$ según Duncan (10)

Se evidenció también que los tratamientos que presentaron menores valores de $\mathrm{pH}$ tienen la mayor producción de ácido láctico, lo que produjo diferencias $(\mathrm{P}<0,05)$ en este indicador entre tratamientos. Se conoce que el $\mathrm{pH}$ de un cultivo cambia con la actividad metabólica y la capacidad amortiguadora del sustrato (17) por diferentes razones, entre las que se encuentran: la liberación de ácidos orgánicos, como el acético y el láctico, por la oxidación incompleta del sustrato, o el consumo de iones amonio, que provocan un descenso en la concentración de hidrogeniones $(\mathrm{pH})$, mientras que la asimilación de los ácidos del medio o la liberación del amonio, debido a la 
desaminación de la urea u otras aminas, inducen el efecto contrario (18).

La disminución que se observó en el pH a las 48 $\mathrm{h}$ de fermentación se relaciona con el incremento que se produjo en la concentración de ácido láctico. Al respecto, Rodríguez (19), al analizar estas variables, encontró correlaciones altamente significativas. Por otra parte, la inclusión de urea incrementó la concentración de amoníaco, lo que pudo afectar directamente el $\mathrm{pH}$; sin embargo, la concentración de ácido láctico y de los AGCC, producto de la hidrólisis de los carbohidratos (monosacáridos) de fácil fermentación presentes en la harina de frutos del árbol del pan, pudieran neutralizar el $\mathrm{pH}(20,21,22$ y 2); por lo tanto, se infiere que en los procesos de FES del fruto del árbol del pan, a medida que se incrementa el nivel de urea, aumenta la concentración de amoníaco $\mathrm{y}$, por ende, el $\mathrm{pH}$, lo que depende, en menor o mayor grado, de la concentración de los ácidos orgánicos (AGCC y láctico).

El amoníaco aumentó en la medida que transcurrió el tiempo de fermentación y se incrementaron los niveles de urea, excepto para el $0,5 \%$ de urea, que no mostró variación en el tiempo. Los mayores valores se encontraron con la adición de 1,5\% de urea, a partir de las $12 \mathrm{~h}$ sin variación hasta las 48 h. Esta variable fue superior a la que reportan Elías y Lezcano (3) en la FES de la caña de azúcar con $1,5 \%$ de urea y diferentes inclusiones de maíz ( 0 , 10,20 y $30 \%$ ). Lo anterior pudiera atribuirse a la hidrólisis parcial o total de la urea a amoníaco (11, $23,24,25)$, por la posible acción de la ureasa de las bacterias ureolíticas encontradas en estos procesos de FES $(23,24)$, lo que posibilita que el amoníaco se pueda utilizar como fuente de nitrógeno, por la biomasa microbiana que se establece en el sistema (26); a la naturaleza del sustrato, y al contenido de humedad que podría afectar el proceso.

Por su parte, Elías et al. (11), al incluir diferentes proporciones de urea $(0,0,5,1,0$ y $1,5 \%)$ en la FES de la caña de azúcar, encontraron que a medida que se incrementó la urea aumentó la concentración de amoníaco y el $\mathrm{pH}$, independiente de la fecha de corte. Elías y Lezcano (25), al estudiar distintas proporciones de nitrógeno aportado por la urea y por la harina de soya desgrasada y sin desgrasar, observaron que al incrementar el nitrógeno, aumentó la concentración de amoníaco y el pH. Similares resultados obtuvieron Rodríguez et al. (27) al determinar el efecto de la urea $(0,5,1,0$ y $1,5 \%$ ) en la FES de mezcla de caña y boniato (Ipomea batata Lam.).

La fermentación de los carbohidratos produce AGCC y otros ácidos orgánicos, pero no en cantidades suficientes como para neutralizar totalmente las producciones de $\mathrm{NH}_{3}$. Se comprobó que en la FES de la harina de frutos del árbol del pan, independientemente del nivel de urea que se empleó, se produjo, casi en su totalidad, ácido acético, como es común en las fermentaciones $(28,29,30$ y 31$)$.

El ácido acético, en ciertas condiciones, inhibe el crecimiento microbiano e induce a la muerte celular de las levaduras (26 y 32). A pH bajo, el ácido acético presente se encuentra principalmente en forma no disociada y puede entrar a la célula microbiana por difusión pasiva (33); en el citoplasma se disocia, debido al $\mathrm{pH}$ más neutro, y se liberan los protones, que bajan el $\mathrm{pH}$ del citoplasma, lo que interfiere con algunos senderos metabólicos (34, 35 y 36), así como en el transporte de nutrientes e iones, en el cambio en la estructura de la membrana, en los ácidos grasos y en la composición de los fosfolípidos, y en la síntesis de proteína.

Referente a la composición química, no hubo interacción entre el nivel de urea añadido y el tiempo de fermentación para los siguientes indicadores: MS, MSR, PV y relación (PV:PB)*100, no así para la PB. En el estudio hubo una disminución en la MS $(P<0,001)$ a medida que transcurrió el tiempo de fermentación. Los niveles de urea no tuvieron incidencia en su comportamiento (Tabla II). Algo similar sucedió con la MSR, la que disminuyó $(P<0,05)$ en 2,34 y 2,94 unidades porcentuales a las 24 y $48 \mathrm{~h}$, respectivamente, con respecto a la hora cero; sin embargo, los niveles de urea no tuvieron efecto sobre este indicador. 
Tabla II. Efecto de niveles de urea y el tiempo de fermentación en la concentración de MS y MSR durante la FES de la HFP

\begin{tabular}{ccc}
\hline Factor & MS, \% & MSR, \% \\
\hline Tiempo, h & $36,31^{\mathrm{c}}$ & $93,57^{\mathrm{c}}$ \\
0 & $35,23^{\mathrm{b}}$ & $92,24^{\mathrm{bc}}$ \\
12 & $34,63^{\mathrm{ab}}$ & $91,83^{\mathrm{ab}}$ \\
24 & $33,68^{\mathrm{a}}$ & $90,63^{\mathrm{a}}$ \\
48 & 0,36 & 0,50 \\
EE \pm & $\mathrm{P}=0,0001$ & $\mathrm{P}=0,0019$ \\
Signif. & & \\
Niveles de urea, \% & 35,44 & 92,52 \\
0 & 35,17 & 91,96 \\
0,5 & 34,76 & 92,02 \\
1,0 & 34,47 & 91,77 \\
1,5 & 0,36 & 0,50 \\
EE \pm & $\mathrm{P}=0,2463$ & $\mathrm{P}=0,7539$ \\
Signif. & $c 0 l u m n a$ & $05 \mathrm{segun}$
\end{tabular}

a, b, c Medias con letras distintas en cada columna difieren a $\mathrm{P}<0,05$ según Duncan (1955)

Las reducciones de MS en el proceso de FES de la HFP pueden deberse, fundamentalmente, a la utilización de los carbohidratos solubles (sacarosa, glucosa, fructosa) de la HFP-FES como fuentes energéticas de los procesos metabólicos, aunque no se descarta que pudo producirse una pérdida de los metabolitos intermediarios, que son volátiles a la temperatura a las que se someten las muestras durante el proceso de secado para este análisis $\left(60{ }^{\circ} \mathrm{C}\right)$; además, pueden deberse a la fermentación del almidón, que predomina en la HFP, según Leyva (2), y también a la hidrólisis de la urea y, posiblemente en menor grado, a la desaminación de péptidos y aminoácidos con producción de amoniaco, los cuales se producen por la acción de los microorganismos presentes en el ecosistema durante sus procesos metabólicos para síntesis celular, oxidándolos a compuestos volátiles, como los ácidos grasos, $\mathrm{CO}_{2}$ y agua, como productos finales (23 y 24). Así, parte del agua que se produce durante la hidrólisis de las moléculas de carbohidratos pudiera evaporarse por el calor metabólico que se genera durante el proceso de la FES (37 y 38).

La reducción de la MS pudo provocar un efecto de concentración relativa del resto de los indicadores que se expresaron en valores porcentuales respecto a ella, tales como la PB, la FDN, la FDA, la MO y la hemicelulosa. La diferencia entre los valores de la PB fue la esperada, de acuerdo con el nivel de urea que se añadió (25). El aumento de los tenores de PB que ocurrieron en la última etapa de la fermentación, independientemente del nivel de urea añadido, pudiera relacionarse con que en ese momento se produjeron mayores retenciones de $\mathrm{NH}_{3}$, hecho que se acentuó con la disminución de los valores de $\mathrm{pH}$, que se obtuvieron durante el proceso, así como con la síntesis de proteína, lo que se refleja en el aumento de la relación $(\mathrm{PV}: \mathrm{PB}) * 100$ al incrementarse los niveles de urea en la misma tabla.

El contenido de PB se muestra en la Tabla III. En el tratamiento control, la PB se mantuvo sin variación, sin embargo, la incorporación de niveles de urea y el aumento del tiempo de fermentación trajo consigo un incremento en la PB. A las 12 y $24 \mathrm{~h}$, con $1,5 \%$ de urea, hubo un incremento en su concentración con respecto a la hora cero, sin diferencia entre ellos. A las $48 \mathrm{~h}$ hubo un aumento mayor de 2,15 unidades porcentuales en la $\mathrm{PB}$, con respecto a la hora cero, al añadir 1,5\% de urea. 
Tabla III. Efecto de niveles de urea y el tiempo de fermentación en la concentración de PB durante la FES de la HFP

\begin{tabular}{lcccccc}
\hline Indicador & Tiempo & \multicolumn{4}{c}{ Niveles de urea, \% } & EE \pm \\
\cline { 2 - 5 } & $\mathbf{h}$ & $\mathbf{0}$ & $\mathbf{0 , 5}$ & $\mathbf{1 , 0}$ & $\mathbf{1 , 5}$ & Signif. \\
\hline & 0 & $5,21^{\mathrm{a}}$ & $8,54^{\mathrm{b}}$ & $11,95^{\mathrm{d}}$ & $14,26^{\mathrm{f}}$ & \\
$\mathbf{P B}, \mathbf{\%}$ & 12 & $5,26^{\mathrm{a}}$ & $8,88^{\mathrm{bc}}$ & $12,55^{\mathrm{de}}$ & $15,42^{\mathrm{g}}$ & 0,23 \\
& 24 & $5,29^{\mathrm{a}}$ & $8,94^{\mathrm{bc}}$ & $12,77^{\mathrm{e}}$ & $15,85^{\mathrm{gh}}$ & \\
& 48 & $5,28^{\mathrm{a}}$ & $9,42^{\mathrm{c}}$ & $12,73^{\mathrm{e}}$ & $16,41^{\mathrm{h}}$ & $\mathrm{P}=0,0142$ \\
\hline
\end{tabular}

$\mathrm{a}, \mathrm{b}, \mathrm{c}, \mathrm{d}, \mathrm{e}, \mathrm{f}, \mathrm{g}, \mathrm{h}$ Medias con letras distintas indican diferencias a $\mathrm{P}<0,05$ según Duncan (1955)

El incremento en el contenido de PB a las 12, 24 y $48 \mathrm{~h}$ de fermentación, al incorporar $1,5 \%$ de urea, pudiera relacionarse con la pérdida de MS, ya que la PB pudiera concentrarse y, por lo tanto, en términos relativos se incrementó; similares resultados encontraron Rodríguez et al. (27) al estudiar diferentes niveles de urea $(0,5,1,0$ y $1,5 \%)$ y tiempos de fermentación $(0,48,72$ y 96 h).
La Tabla IV presenta los resultados de PV y la relación $(\mathrm{PV}: \mathrm{PB}) * 100$. El mayor contenido de PV se obtuvo a las 24 y $48 \mathrm{~h}$, sin diferencia entre ellos, en cuanto al factor tiempo. Al añadir urea al sistema hubo diferencias entre todos los tratamientos en estudio, con un incremento en su contenido al incorporar $1,5 \%$ de urea.

Tabla IV. Efecto de niveles de urea y del tiempo de fermentación en la concentración de PV y en la relación (PV:PB)*100 durante la FES de la HFP

\begin{tabular}{lll}
\hline Factor & $\mathbf{P V}, \mathbf{\%}$ & $\mathbf{( P V : P B})^{*} \mathbf{1 0 0}$ \\
\hline Tiempo, h & & \\
0 & $6,39^{\mathrm{a}}$ & 60,69 \\
12 & $6,67^{\mathrm{ab}}$ & 60,38 \\
24 & $7,03^{\mathrm{bc}}$ & 63,78 \\
48 & $7,19^{\mathrm{c}}$ & 63,72 \\
$\mathbf{E E} \pm$ & 0,15 & 1,30 \\
Signif. & $\mathrm{P}=0,0014$ & $\mathrm{P}=0,1194$ \\
Niveles de urea, \% & & \\
0 & $2,78^{\mathrm{a}}$ & $52,97^{\mathrm{a}}$ \\
0,5 & $5,12^{\mathrm{b}}$ & $57,31^{\mathrm{b}}$ \\
1,0 & $8,48^{\mathrm{c}}$ & $67,89^{\mathrm{c}}$ \\
1,5 & $10,90^{\mathrm{d}}$ & $70,39^{\mathrm{c}}$ \\
EE \pm & 0,15 & 1,30 \\
Signif. & $\mathrm{P}<0,0001$ & $\mathrm{P}<0,0001$ \\
\hline
\end{tabular}

a, b, c, d Medias con letras distintas en cada columna difieren a $\mathrm{P}<0,05$ según Duncan (1955)

En cuanto a la relación (PV:PB)*100, aumentó su concentración en la medida en que se añadió urea al sistema; hubo un aumento de 17,42 unidades porcentuales al añadir $1,5 \%$ de urea respecto al tratamiento control $(0 \%)$; sin embargo, al transcurrir el tiempo de fermentación se mantuvo sin variación entre tratamientos. 
En el presente estudio, el aumento en el contenido de PV se produjo a las 24 y $48 \mathrm{~h}$ de fermentación $y$ al adicionar urea, de lo que infiere que los carbohidratos fácilmente fermentables y el nitrógeno ureico se utilizaron en la formación de protoplasma celular. Esto demuestra la importancia de la adición de una fuente de NNP, como la urea, para la síntesis de proteína microbiana en procesos de FES, como plantearon Elías et al. (11). Thompson y Hobson (40) indicaron que cuando la disponibilidad de amoníaco es limitante para los microorganismos, ellos entran a fase de latencia y fermentan los azúcares disponibles, con producción de AGV.

En parte, este incremento de PV a las 24 y 48 $h$ se puede relacionar también con el proceso de concentración relativa que ocurre durante esos tiempos de fermentación para la PB en su transformación en PV. Por otro lado, este aumento de PV puede deberse a los senderos metabólicos y enzimas disponibles en el sistema para incorporar el amonio a la célula microbiana como sillares de aminoácidos, los que dependen de la concentración de amonio existente. Según Elías y Lezcano (26), la eficiencia con la que el NNP de la urea se convierte en NP varía en relación con los sustratos empleados y el manejo, pero sobre todo con la disponibilidad de energía y las fuentes de carbono (carbohidratos solubles, almidón y grasas), aminoácidos, péptidos, vitaminas y minerales, y que el nivel de urea óptimo depende del tipo de sustrato a fermentar.

Los valores de PV son similares a los que reportan $(3,25,27,28,41$ y 42$)$; sin embargo, son menores a los que informa (31), cuando añade al tubérculo de yuca en FES $2 \mathrm{~g}$ de urea en todos los tratamientos y carbonato de calcio para controlar el pH. Esto confirma que a bajo $\mathrm{pH}$ hay inhibición de la síntesis de proteína, por el efecto letal que pueden producir algunos ácidos orgánicos como el acético, lo que se corrobora en la Tabla 4 donde con el aumento de los niveles de urea hubo mayor síntesis de PV y relación (PV:PB)*100, lo que coincide con los valores de $\mathrm{pH}$ más altos y menores concentraciones de ácido acético.
La relación $(\mathrm{PV}: \mathrm{PB}) * 100$ después de la FES de la HFP es mayor a la que informan Elías y Lezcano (3) en el Sacchamaíz, y Rodríguez et al. (39 y 41) en la dinámica de fermentación del Sacchaboniato hasta las $72 \mathrm{~h}$, y menores a las que estos últimos investigadores obtuvieron a las $96 \mathrm{~h}$.

Se concluye que la adición de urea en el proceso de fermentación de la harina de frutos del árbol del pan en estado sólido incrementa la síntesis de proteína microbiana.

\section{Agradecimientos}

A CAPES (Brasil), por su apoyo financiero, y al Instituto de Ciencia Animal (ICA) y a la Universidad de Guantánamo, por la formación.

\section{Referencias}

(1) Brea, O.; Elías, A.; Ortiz, A.; Herrera, F. y Motta, W. Utilización de la harina de frutos del árbol del pan (Artocarpus altilis) fermentada en estado sólido, en dietas destinadas a cerdos en preceba. Rev. Cubana de Cien. Agríc. 2014; 48(4): 391-398.

(2) Leyva, C. Caracterización química de harinas de frutos y hojas del árbol del pan (Artocarpus altilis) y su empleo en la alimentación de pollos, conejos y ovinos de ceba. Tesis de Doctorado. Instituto de Ciencia Animal. Cuba. 2010.

(3) Elías, A. y Lezcano, O. Efecto de la inclusión de niveles de harina maíz en la fermentación de la caña de azúcar. Rev. Cubana de Cienc. Agríc. 1994; 28(2): 319.

(4) Ramos, J.A.; Elías, A. y Herrera, F. R. Procesos para la producción de un alimento proteico-energético para animales. Efecto de cuatro fuentes energéticas en la fermentación en estado sólido (FES) de la caña de azúcar. Rev. Cubana de Cien. Agríc. 2006; 40(2): 51.

(5) Becerra, A.; Rodríguez, C.; Jiménez, J.; Ruiz, O.; Elías A. y Ramírez, A. Urea y maíz en la fermentación aeróbica de bagazo de manzana 
para la producción de proteína. Tecnociencia Chihuahua. 2008; 2(1): 7-14.

(6) Elías, A. y Herrera, F. R. Producción de alimentos para animales a través de procesos biotecnológicos sencillos con el empleo de microorganismos beneficiosos activados (MEBA). VITAFERT. 2008. 8-13.

(7) Díaz, B. Evaluación de residuos agrícolas poscosecha en ensilajes inoculados con preparados microbianos nativos para la alimentación de vacas lecheras en Ecuador. Tesis de Doctor en Ciencias Veterinarias. Instituto de Ciencia Animal. La Habana, Cuba. 2014.

(8) Leyva, C. y Valdivié, M. Fruta del pan y la alimentación alternativa en animales de traspatio. Rev Asociación Cubana de Producción Animal. 2007. 1:48.

(9) Di Rienzo, J.A.; Casanoves, F.; Balzarini, M.G.; González, L.; Tablada, M. y Robledo C.W. InfoStat versión 2012. Grupo InfoStat, FCA, Universidad Nacional de Córdoba, Argentina. http://www.infostat.com.ar.

(10) Duncan, B. Multiple Range and Multiple F Test. Biometrics, 1955; 11: 1-7. DOI: http:// dx.doi.org/10.2307/3001478.

(11) Elías, A.; Lezcano, O.; Lezcano, P.; Cordero, J. \& Quintana, L. Una reseña descriptiva sobre el desarrollo de una tecnología de enriquecimiento proteico de la caña de azúcar a través de fermentación en estado sólido. Saccharina. Rev. Cubana de Cienc. Agríc. 1990; 24:1.

(12) Conway, E.J. Microdiffusion analysis and volumetric error, $4^{\text {th }}$ ed. Crosby Lockwood and Sons, Ltd. London. 1957.

(13) Erwin, E.S.; Marco, G.J. y Emery, E.M. Volatile fatty acid analysis of blood and rumen fluid by gas chromatography. J. Dairy Sci. 1961. 44: 1768-1776. DOI: http://dx.doi.org/10.3168/jds. S0022-0302(61)89956-6.
(14) Madrid, J.; Martínez Teruel, A.M.; Hernández, F. y Megias, M. D. Comparative study on the determination of lactic acid in silage juice by colorimetric, high performance liquid chromatography and enzymatic methods. Journal of the Science of Food and Agriculture. 1999; 79: 1722-172. DOI: http://dx.doi.org/10.1002/ (SICI) 1097-0010(199909)79:12<1722::AID -JSFA427> 3.0.CO;2-S.

(15) AOAC. Official Methods of Analysis. 17th Ed. Assoc. Off, Agric. Anal. Chem. Arlington, Virginia. 2000.

(16) Meir, H. Laborpractike. Tierernahrung und futtermittel kunde fur Tierproduktion. Verlag, Germany. 1986, pp. 35-40.

(17) Sahlin, P. Fermentation as a method of food processing. Production of organic acids, $\mathrm{pH}$-devolopment and microbial growth in fermenting cereals. Licentiate thesis. Lund Institute of Technology. Lund University. 1999.

(18) Prior, B.; Jame, C.; Preez, D. y Reir, P.W. Enviroment parameters solid productos finales de la fermentación para la obtención de cuatro tipos de Sacarinas Rabbit Congress, 6, Touluse, França. 1992. 1: 271-276.

(19) Rodríguez, Z. Uso del boniato (Ipomea batata Lam.) en la tecnología de fermentación en estado sólido de la caña de azúcar (Saccharum officinarum). Tesis de Doctor en Ciencias. Instituto de Ciencia Animal, Habana, Cuba. 2004.

(20) Ramos, J.A.; Elías, A. y Herrera, F. R. Procesos para la producción de un alimento proteico-energético para animales. Efecto de cuatro fuentes energéticas en la fermentación en estado sólido (FES) de la caña de azúcar. Revista Cubana de Cienc. Agríc. 2009; 43(1): 51-54.

(21) Ramos, J. A.; Aranda I., E. M. y Elías, A. Patrones de fermentación ruminal y digestibilidad in situ en bovinos alimentados con forraje $y$ suplementos a base de caña de azúcar fermentada en estado sólido. Producción animal tro- 
pical en II Congreso Internacional de Producción Animal, La Habana, Cuba. 2007.

(22) Cárdenas, J. R.; Aranda, E.M.; Hernández, D.; Lagunes, L. del C.; Ramos, J.A. y Salgado, S. Obtención de un alimento fermentado en estado sólido a partir del bagacillo de retorno, pulido de arroz e inóculos. Su utilización en la alimentación animal. Revista Cubana de Cienc. Agríc., 2008; 42(2): 173-176.

(23) Valiño, E.; Elías, A., Álvarez, E., Quintana, M. \& Montes de Oca, N. Composición de especies de bacterias aisladas del proceso de la Saccharina. 1. Bacteria Gram negativas. Rev. Cubana de Cienc. Agríc. 1994a; 28(1):69-74.

(24) Valiño, E.; Elías, A.; Álvarez, E.; Quintana, M. y Montes de Oca, N. Composición de especies de bacterias aisladas del proceso de obtención de la Saccharina. II. Bacterias gram positivas. Rev. Cubana Cienc. Agríc. 1994b; 28 (1): 7580.

(25) Elías, A. y Lezcano, O. Inclusión de niveles de harina de soya desgrasada y sin desgrasar en la fermentación de la caña de azúcar en estado sólido (Sacchasoya). Rev. Cubana Cienc. Agríc. 2000; 34(2): 143-150.

(26) Elías, A. y Lezcano, O. Efecto de la fuente de $\mathrm{N}$ y algunos factores de crecimiento en la población de levaduras que se establecen en la producción de Saccharina. Rev. Cubana Cienc. Agríc. 1993; 28(3): 319-325.

(27) Rodríguez, Z.; Boucourt, R.; Elías, A. y Núñez, O. Efecto de los niveles de nitrógeno ureico en la síntesis proteica durante la fermentación de mezcla de caña (Saccharum officinarum) y boniato (Ipomea batata Lam.). Revista Cubana Cienc. Agríc. 2001a; 35(1): 29-36.

(28) Lezcano, O. y Elías A. Efecto de la temperatura y la urea en la fermentación de la caña de azúcar para producir Saccharina. Rev. Cubana Cienc. Agrí́c. 1992; 26(3): 291-294.
(29) Carrasco, E.; Boucourt, R.; Elías, A. y Febles, I. Nivel de urea y grosor de la capa en los indicadores fermentativos de la caña de azúcar procesada con excreta vacuna. Revista Cubana Cienc. Agríc. 1998b; 32(4): 395-399.

(30) Rodríguez, Z. Uso del boniato (Ipomea batata Lam.) en la tecnología de fermentación en estado sólido de la caña de azúcar (Saccharum officinarum). Tesis de Doctor en Ciencias. Instituto de Ciencia Animal, Habana, Cuba. 2004.

(31) Rodríguez, Y. Obtención de un alimento energético proteico a través de la FES de la caña de azúcar y el tubérculo de yuca. Tesis de Master en Ciencias Veterinarias. Universidad Agraria de la Habana. Instituto de Ciencia Animal. Habana, Cuba. 2005.

(32) Ludovico, P.; João, S.M.; Silva, M.T.; Leão, C. y Côrte-Real. Saccharomyces cerevisiae commits to programmed cell death process in response to acetic acid. Microbiol Rev. 2001; 147: 2409-2415. DOI: http://dx.doi. org/10.1099/00221287-147-9-2409.

(33) Geros, H.; Cassio, F. y Leao, C. Utilization and transport of acetic acid in Dekker aanomala and their implications on the survival of the yeast in acidic environments. J. Food Prot. 2000; 63: 96-101.

(34) Roe, A.J.; O 'Byrne, C.; McLaggan, D. y Booth I.R. Inhibition of Escherichia coly growth by acetic acid: a problem with methionine biosynthesis and homocy steine toxicity. Microbiol Rev. 2002; 148, 2215-2222.

(35) Augstein, A.; Barth, K.; Gentsch, M.; Kohlwein, S.D. y Barth, G. Characterization, localization and functional analysis of Gpr1p, a protein affecting sensitivity to acetic acid in the yeast Yarrowiali polytica. Microbiol Rev. 2003. 149: 586-600.

(36) Hazan, R.; Levine, A. y Abeliovich, H. Benzoic acid, a weak organic acid food preservative, exerts specific effects on intracellular membra- 
ne trafficking pathways in Saccharomyces cerevisiae. Applied and Environmental Microbiology. 2004; 70(8): 4449-4457. DOI: http://dx.doi. org/10.1128/AEM.70.8.4449-4457.2004.

(37) Pandey, A.; Soccol C.R.; Rodríguez-León, J.A. y Nigam, P. Solid-state fermentation in biotechnology. Fundamentals and applications. Asia Tech Publishers. 2001.

(38) Krishna, C. Critical Solid-State Fermentation Systems-An Overview Reviews in Biotechnology; ProQuest Agriculture Journals. 2005.

(39) Rodríguez, Z.; Elías, A.; Boucourt, R. y Núñez, O. Dinámica de fermentación de mezclas de caña (Saccharum officinarum) y boniato (Ipomea batata Lam.). Revista. Cubana Cienc. Agríc. 2001b; 35(2): 147-151.
(40) Thompson, J.K. y Hobson, P.N. Polysaccharide synthesis and degradation by rumen microorganisms in vitro. J. Agric. Sci. Camb. 1971; 76: 423-432. DOI: http://dx.doi.org/10.1017/ S0021859600069409.

(41) Rodríguez, Z.; Elías, A. y Riveri Z. Estudio de utilización de boniato (Ipomea batatas Lam) en la fermentación en estado sólido de la caña de azúcar. Revista. Cubana Cienc. Agríc. 1998; 32(3): 307-312.

(42) Caraveo, A. Efecto de los niveles de urea en la caña fermentada con pulidura de arroz. Sacchapulido. Tesis presentada en opción al Título de Master en Ciencias. 2008. p. 21-29. 